\title{
Loss of Villin Immunoexpression in Colorectal Carcinoma Is Associated with Poor Differentiation and Survival
}

\author{
Jaudah Al-Maghrabi, ${ }^{1,2,3}$ Wafaey Gomaa, ${ }^{2,4}$ Abdelbaset Buhmeida, ${ }^{3}$ \\ Mohmmad Al-Qahtani, ${ }^{3}$ and Mahmoud Al-Ahwal ${ }^{1,5}$ \\ ${ }^{1}$ Scientific Chair for Colorectal Cancer, King Abdulaziz University, P.O. Box 80205, Jeddah 21589, Saudi Arabia \\ ${ }^{2}$ Department of Pathology, King Abdulaziz University, P.O. Box 80205, Jeddah 21589, Saudi Arabia \\ ${ }^{3}$ Center of Excellence in Genomic Medicine Research, King Abdulaziz University, P.O. Box 80205, Jeddah 21589, Saudi Arabia \\ ${ }^{4}$ Department of Pathology, Faculty of Medicine, Minia University, El Minia, Egypt \\ ${ }^{5}$ Department of Medicine, King Abdulaziz University, P.O. Box 80205, Jeddah 21589, Saudi Arabia
}

Correspondence should be addressed to Jaudah Al-Maghrabi; jalmaghrabi@hotmail.com

Received 9 June 2013; Accepted 29 June 2013

Academic Editors: A. Amedei and A. J. Karayiannakis

Copyright @ 2013 Jaudah Al-Maghrabi et al. This is an open access article distributed under the Creative Commons Attribution License, which permits unrestricted use, distribution, and reproduction in any medium, provided the original work is properly cited.

Background and Aims. Villin is a highly specialised protein and is expressed in intestinal and renal proximal tubular epithelium. It was detected in colorectal carcinomas (CRC) and other nongastrointestinal tumours. The aim of the current study is to investigate the immunohistochemical expression of villin in a subset of primary CRC and determine its relation to tumour differentiation, invasion, nodal metastasis, recurrence, and disease-free survival. Patients and Methods. Paraffin blocks of 93 cases of CRC were retrieved constituting 93 primary CRC and 58 adjacent normal mucosa. Immunohistochemistry was performed using antivillin antibody. The extent (\%) of villin immunoexpression was categorised for statistical analysis. Statistical tests were used to determine the association of villin with clinicopathological characteristics: age, sex, tumour location, tumour size, depth of invasion, tumour grade, nodal metastasis, lymphovascular invasion, margin status, recurrence, and survival. Results. Villin immunostaining results showed that villin is downregulated in CRC. Villin has no association with age, sex, tumour location, depth of invasion, nodal metastasis, lymphovascular invasion, margin status, and recurrence. However, villin is expressed in higher rate in CRC less than $5 \mathrm{~cm}$, well- and moderately differentiated CRC. Poor survival was associated with tumour with low villin immunoexpression. Conclusion. Villin was downregulated in CRC. Villin immunoexpression in CRC is associated with better survival, well-differentiated tumours, and small-sized tumours. Villin has no significant association with disease recurrence or nodal metastasis. More in vivo and in vitro studies are required for further elucidation of how villin may be involved in CRC.

\section{Introduction}

Although 70\% of colorectal carcinoma (CRC) cases undergo a curative surgery, $50 \%$ of surgically cured patients will have at a time an advanced local recurrence or metastases [1]. CRC is a common type of cancer with a considerably poor prognosis and mortality all over the world. The extent of local invasion and tumour metastasis are important factors to determine disease outcome. Distant metastases aggravate treatment failure, and patients will be subjected to palliative treatment. Molecular markers of metastasis are essential to improve treatment protocols $[1,2]$. Understanding the molecular pathology underlying CRC needs continuous efforts to discover more prognostic molecules to predict disease outcome and improve therapeutic interventions. The Saudi Arabian National Cancer Registry in 2005 showed that CRC represented $5.3 \%$ of all tumours. CRC is the most common malignancy in Saudi males, while in females, CRC is the third most common malignancy and represented $4 \%$ of all tumours [3].

Villin is a protein that belongs to the gelsolin family of calcium-regulated actin-binding proteins [4]. It was first isolated and characterised in the microvilli of intestinal epithelium [5]. Hence, the name villin came from. Microvilli are located on the apical membrane (brush border) of polarised epithelial cells and increase the surface area for the 
TABLE 1: Clinicopathological characteristics of cases.

\begin{tabular}{|c|c|}
\hline Item & Number (\%) \\
\hline \multicolumn{2}{|l|}{ Age } \\
\hline$<60$ years & $45 / 93(48.4 \%)$ \\
\hline$\geq 60$ years & $48 / 93(51.6 \%)$ \\
\hline \multicolumn{2}{|l|}{ Sex } \\
\hline Male & $44 / 93(47.3 \%)$ \\
\hline Female & $49 / 93(52.7 \%)$ \\
\hline \multicolumn{2}{|l|}{ Tumour location } \\
\hline Right colon & $30 / 93(32.2 \%)$ \\
\hline Left colon & $26 / 93(28 \%)$ \\
\hline Rectum & $37 / 93(39.8 \%)$ \\
\hline \multicolumn{2}{|l|}{ Tumour size } \\
\hline$<5 \mathrm{~cm}$ & $29 / 93(31.2 \%)$ \\
\hline$\geq 5 \mathrm{~cm}$ & $24 / 93(25.8 \%)$ \\
\hline Data not applicable & $40 / 93(43 \%)$ \\
\hline \multicolumn{2}{|l|}{ Primary tumour } \\
\hline $\mathrm{T} 1$ & $0 / 93(0 \%)$ \\
\hline $\mathrm{T} 2$ & $12 / 93(12.9 \%)$ \\
\hline T3 & $10 / 93(10.8 \%)$ \\
\hline $\mathrm{T} 4$ & $31 / 93(33.3 \%)$ \\
\hline Data not applicable & $40 / 93(43 \%)$ \\
\hline \multicolumn{2}{|l|}{ Tumour grade } \\
\hline Well differentiated & $25 / 93(26.9 \%)$ \\
\hline Moderately differentiated & $63 / 93(67.7 \%)$ \\
\hline Poorly differentiated & $5 / 93(5.4 \%)$ \\
\hline \multicolumn{2}{|l|}{ Nodal metastasis } \\
\hline Positive & $31 / 93(33.3 \%)$ \\
\hline Negative & $24 / 93(25.8 \%)$ \\
\hline Data not applicable & $38 / 93(40.9 \%)$ \\
\hline \multicolumn{2}{|l|}{ Lymphovascular invasion } \\
\hline Positive & $7 / 93(7.5 \%)$ \\
\hline Negative & $46 / 93(49.5 \%)$ \\
\hline Data not applicable & $40 / 93(43 \%)$ \\
\hline \multicolumn{2}{|l|}{ Margin status } \\
\hline Involved & $6 / 93(6.5 \%)$ \\
\hline Free & $47 / 93(50.5 \%)$ \\
\hline Data not applicable & $40 / 93(43 \%)$ \\
\hline \multicolumn{2}{|l|}{ Recurrence } \\
\hline Recurrence & $30 / 93(32.25 \%)$ \\
\hline No recurrence & $33 / 93(35.5 \%)$ \\
\hline Data not available & $30 / 93(32.25 \%)$ \\
\hline \multicolumn{2}{|l|}{ Status at end point } \\
\hline Died of disease & $23 / 93(24.7 \%)$ \\
\hline Alive & $70 / 93(75.3 \%)$ \\
\hline
\end{tabular}

T1: tumour invades submucosa; T2: tumour invades muscularis propria; T3: tumour invades through the muscularis propria into the subserosa or into nonperitonealised pericolic or perirectal tissues; $\mathrm{T} 4$ : tumour directly invades other organs or structures and/or perforates visceral peritoneum.

absorption of nutrients. Microvilli are sustained by bundles of parallel actin filaments that are organised by multiple actin-binding proteins including villin [6]. Villin constitutes a major component of the brush border cytoskeleton. It functions in the capping, severing, and bundling of actin filaments $[4,7]$. Villin is a highly specialised protein and specifically expressed in intestinal epithelium and renal proximal tubules. Villin had been detected in CRC and has been used to differentiate neoplasms of intestinal origin from nonintestinal neoplasms $[8,9]$. Villin is also expressed in gastrointestinal neuroendocrine tumours [10].

As the outcome of CRC is largely dependent on the extent of local and metastatic tumour spread, the identification of molecular markers of metastatic clones is essentially needed for developing new therapies in CRC. The aim of the current study is to investigate the immunohistochemical expression of villin in a subset of primary CRC and determine its relation to tumour differentiation, invasion, nodal metastasis, and recurrence.

\section{Patients and Methods}

The study included 93 cases of CRC (in the period from 2003 to 2009) that were retrieved from the archives of the Department of Pathology at King Abdulaziz University, Jeddah, Saudi Arabia. Table 1 shows the clinicopathological characteristics of this subset of patients. Age ranges from 24 to 91 years (median 60 years). The study was approved by the Research Committee of the Biomedical Ethics Unit, Faculty of Medicine, King Abdulaziz University.

2.1. Immunostaining of Villin. Four $\mu \mathrm{m}$ thick sections were prepared from paraffin blocks and mounted on positive charged slides (Leica Microsystems Plus Slides, Menzel, Braunschweig, Germany). Sections were deparaffinised in xylene and rehydrated in graded alcohol. Slides were immersed in $\mathrm{H}_{2} \mathrm{O}_{2}(0.3 \%)$ for 12 minutes to block the endogenous peroxidase activity. Slides were then pretreated in microwave oven in $10 \mathrm{mM}$ citrate buffer $(\mathrm{pH} 6)$ for three cycles of 5 minutes each. Anti-human rabbit polyclonal antibody to villin (E1664 from Springer Bioscience) was used at dilution 1:50 with incubation time 30 minutes at room temperature. Immunostaining was carried out in an automated immunostainer (BenchMark XT, Ventana Medical systems Inc., Tucson, AZ, USA) according to the manufacturer's instructions. Subsequently, slides were washed, counterstained with Mayer's haematoxylin, and mounted. Negative control (by substitution of primary antibody with Tris-buffered saline) was used. Normal intestinal mucosa was used as an internal positive control.

2.2. Interpretation of Villin Immunostaining. In order to evaluate villin immunostaining, we first observed the subcellular localisation of villin in normal colonic mucosa adjacent to tumours and in tumour cells as previously described. Three patterns of positive villin staining were observed: a primary brush border pattern (apical membranous), a primary cytoplasmic pattern, and mixed diffuse cytoplasmic pattern with brush border staining which was also observed $[8,9]$. The apical membranous expression and cytoplasmic localisations were scored independently, and the mixed pattern was calculated whenever both patterns were noted together. To assess 
TABLE 2: The incidence of villin immunolocalisation in CRC and normal mucosa.

\begin{tabular}{|c|c|c|c|c|c|}
\hline & \multicolumn{2}{|c|}{ Frequency of expression (positive cases) } & \multicolumn{3}{|c|}{ Pattern of localisation (number (\%)) } \\
\hline & Total & Number $(\%)$ & Brush border & Cytoplasmic & Mixed \\
\hline $\begin{array}{l}\text { Adjacent normal colonic } \\
\text { mucosa }\end{array}$ & 58 & $58(100 \%)$ & $56(96.5 \%)$ & $0(0 \%)$ & $2(3.5 \%)$ \\
\hline CRC & 93 & $79(84.9 \%)$ & $0(0 \%)$ & $23(24.7 \%)$ & $56(60.2 \%)$ \\
\hline
\end{tabular}

Brush border expression: a primary brush border staining only; cytoplasmic expression: a diffuse cytoplasmic expression without any brush border staining; mixed: a primary cytoplasmic staining pattern with minor brush border staining.

the extent (\%) of villin expression (apical membranous and cytoplasmic), all available normal and tumour cells in each section were counted at microscope magnification of 200x. Positive cells were then counted. The mean values of positivity were calculated and expressed as a percentage of the total number of normal and tumour cells. For statistical analysis, we have categorised the extent of villin immunoexpression as negative expression $(0=0 \%)$, low expression $(1=1-9 \%)$, moderate expression $(2=10-50 \%)$, and high expression $(3 \geq$ $50 \%)$.

2.3. Statistical Analysis. Data is presented as the mean \pm standard error of the mean (SEM). Differences between two groups of cases for one variable were tested by using the Mann-Whitney test. To test association between three groups of cases for one independent variable, the KruskalWallis test was used. Difference between expression in normal mucosa and tumour was tested by Wilcoxon signed-rank test. Multivariate logistic regression analysis was used to predict lymph node metastasis and recurrence in relation to immunoexpression of villin. Beta coefficient and 95\% confidence intervals (CI) were denoted for each analysis. The Kaplan-Meier procedure was used to calculate the survival probabilities, and the log-rank test was used to compare the difference between survivals. The end point for patients was death from tumour (disease-specific survival (DSS)). DSS was calculated as the time from diagnosis to death or to the date of last seen alive. Patients who died of causes other than tumour or unknown causes were censored. Statistical procedures were performed using SPSS Release 16.0. Statistical significance was determined at $P$ value of $\leq$ 0.05 , and tests were two sided.

\section{Results}

Basic Data of Villin Immunostaining. We have observed a brush border (apical membranous) with minor cytoplasmic component in $100 \%$ of samples of normal colonic and intestinal crypts (Figure 1(a)). In tumour cells, cytoplasmic immunoexpression was noted in $84.9 \%$ of examined samples. In $60.2 \%$ of samples, there was an associated apical membranous staining, mixed pattern (data is shown in Table 2) (Figures 1(b)-1(d)). A high level of villin protein immunoexpression was observed in normal mucosa adjacent to tumour (100\% of samples; mean 77.84\%; SEM: 3.284) than in tumour samples (84.9\% of cases; mean: 17.32\%; SEM: 1.514); $P<0.001$ (data is presented in Table 2). In normal mucosa, the incidence of negative and low categories of expression was not observed, while high expression constitutes a large number and high extent $P<0.001$. Also in tumour samples, the negative and low expression categories were much lower than moderate category $P<0.001$ (data is shown in Table 3 ).

3.1. Villin Immunoexpression and Clinicopathological Characteristics. The distribution of villin expression in tumour samples in relation to clinicopathological characteristics is presented in Table 4 . There was no statically significant difference in villin immunoexpression in regards to age, sex, tumour location, depth of invasion (primary tumour), nodal metastasis, lymphovascular invasion, margin status, recurrence, or status at end point. However, the mean of villin immunoexpression in well differentiated, and moderately differentiated tumours are higher than in poorly differentiated tumour $(P=0.035)$. Also, the mean of villin immunoexpression was statically higher in tumours measuring less than $5 \mathrm{~cm}$ than in larger tumours $(P=0.042)$.

On multivariate logistic regression analysis, villin immunoexpression showed no statistical significance as an independent predictor of lymph node metastasis (beta coefficient $=1.652$, confidence interval $=1.447-1.857, P$ value $=0.255)$ or of recurrence: (beta coefficient $=1.532$, confidence interval $=1.327-1.737, P$ value $=0.488)$. The Kaplan-Meier survival analyses showed that villin immunoexpression in CRC has a significant association with a more favourable diseasespecific survival (log-rank $=6.604, P=0.037)($ Figure 2).

\section{Discussion}

CRC has a relatively poor prognosis, in which the outcome is determined by the extent of local and metastatic tumour spread. The 5-year survival rate ranges from $90 \%$ in stage I disease to $<10 \%$ in metastatic disease (stage IV) [1]. The molecular pathogenesis of CRC involves transformation of normal colonic epithelium, adenoma, and then carcinoma. This transformation is tightly linked to molecular alterations that lead to abnormal cellular differentiation, proliferation, migration, and apoptosis [11]. Currently, several prognostic factors exist, including clinical staging classification. However, specific markers for metastatic clones in CRC are required to predict the disease outcome and improve the therapeutic intervention.

Villin is an actin regulatory protein that is localised in all the intestinal epithelial cells and gastrointestinal tractassociated exocrine glands [12,13]. Villin regulates intestinal 




(a)

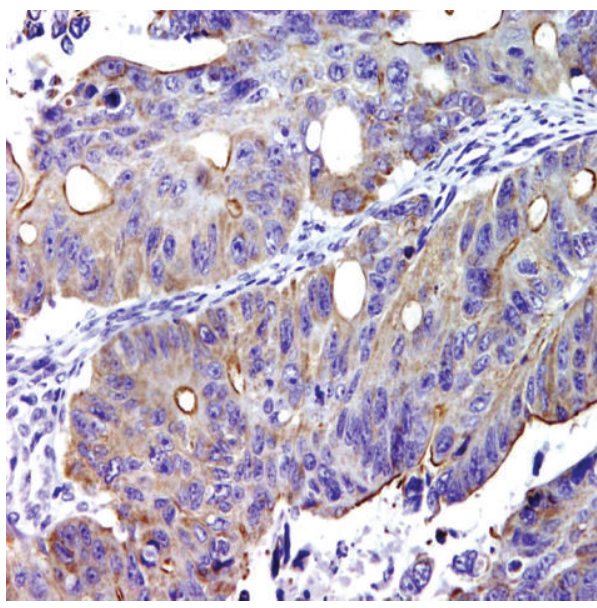

(c)

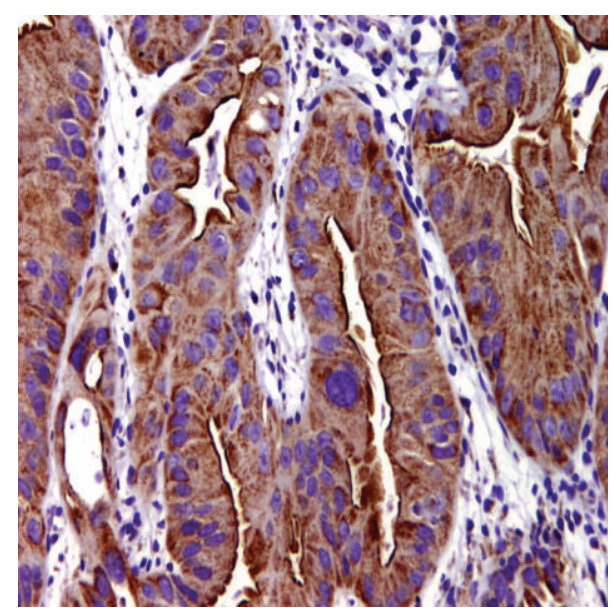

(b)

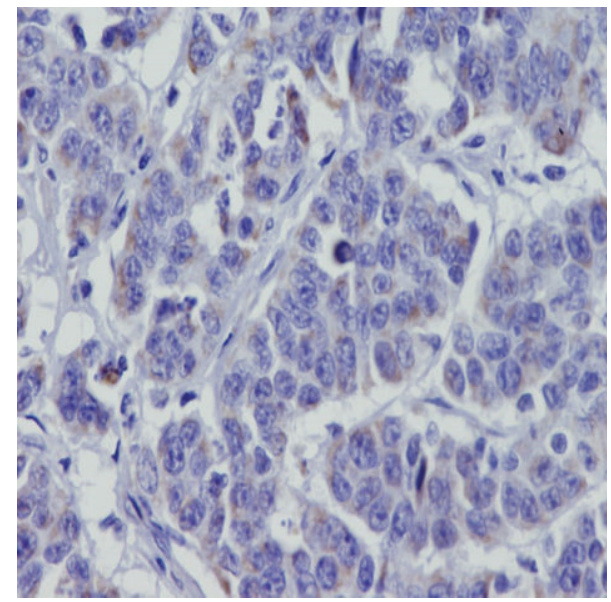

(d)

FIgURE 1: Villin immunoexpression in normal colonic mucosa and in colorectal carcinoma using immunohistochemical labelling with antivillin antibody with diaminobenzidine used as the chromogen and haematoxylin as counterstain. Magnification used was 200x. (a) Villin expression in normal colonic crypts showing a well-organised brush border pattern together with minor cytoplasmic dots in all crypts. (b) Expression in a well-differentiated tumour; a strong staining is shown in both cytoplasm and brush border (mixed pattern). (c) Villin expression in a moderately differentiated colorectal carcinoma; staining is shown in both cytoplasm and brush border (mixed pattern) with less extent and intense staining than in (b). (d) Villin expression in a poorly differentiated colorectal carcinoma showing very minor cytoplasmic staining with no membranous staining.

TABLE 3: Category of villin immunoexpression.

\begin{tabular}{|c|c|c|c|c|c|c|}
\hline \multirow{2}{*}{ Category } & \multicolumn{3}{|c|}{ Normal adjacent mucosa } & \multicolumn{3}{|c|}{ Tumours } \\
\hline & Number (\%) & Mean (SEM) & $P$ value* & Number (\%) & Mean (SEM) & $P$ value ${ }^{*}$ \\
\hline Negative $(0)$ & $0 / 58(0 \%)$ & $0(0)$ & \multirow{4}{*}{$<0.001$} & $14 / 93(15 \%)$ & $0(0)$ & \multirow{4}{*}{$<0.001$} \\
\hline Low (1) & $0 / 58(0 \%)$ & $0(0)$ & & 17/93 (18.3\%) & $5.47(0.73)$ & \\
\hline Moderate (2) & $7 / 58(12.1 \%)$ & $28.57(4.59)$ & & $62 / 93(66.7 \%)$ & $24.16(1.61)$ & \\
\hline High (3) & $51 / 58(87.9 \%)$ & $84.61(2.46)$ & & $0 / 93(0 \%)$ & $0(0)$ & \\
\hline
\end{tabular}

${ }^{*}$ The Kruskal-Wallis test; SEM: standard error of the mean.

epithelial cell morphology, actin reorganisation, and cell motility $[14,15]$. Villin expression in malignant tumour was described first by Moll et al. [9] and Bacchi and Gown [8]. Villin has been also detected in hepatocellular carcinoma (9\%) and showed cytoplasmic staining pattern. In cholangiocarcinoma expression was detected in $20 \%$ of cases and showed only cytoplasmic pattern [16]. In another study, $31 \%$ of HCCs were villin positive. Most displayed a cytoplasmic staining pattern. In $22 \%$ of cholangiocarcinoma cases there was cytoplasmic and apical staining for villin [17]. The expression of the villin protein has been detected in renal cell carcinoma [18]. Accumulation of villin has been observed 
TABLE 4: Distribution of villin-positive immunoexpression in relation to clinicopathological characteristics.

\begin{tabular}{|c|c|c|c|}
\hline & Number (\%) & Mean (SEM) & $P$ value \\
\hline \multicolumn{4}{|l|}{ Age } \\
\hline$>60$ years & $38 / 45(84.4 \%)$ & $16.24(2.13)$ & \multirow[t]{2}{*}{$0.558^{\bigotimes}$} \\
\hline$\geq 60$ years & $41 / 48(85.4 \%)$ & $18.33(2.16)$ & \\
\hline \multicolumn{4}{|l|}{ Sex } \\
\hline Male & $35 / 44(79.5 \%)$ & $15.8(2.26)$ & \multirow{2}{*}{$0.270^{\bowtie}$} \\
\hline Female & $44 / 49(89.8 \%)$ & $18.69(2.04)$ & \\
\hline \multicolumn{4}{|l|}{ Tumour location } \\
\hline Right colon & $25 / 30(83.3 \%)$ & $17.6(2.33)$ & \multirow{3}{*}{$0.891^{*}$} \\
\hline Left colon & $21 / 26(80.8 \%)$ & $16.69(3.16)$ & \\
\hline Rectum & $34 / 37(91.9 \%)$ & $17.54(2.51)$ & \\
\hline \multicolumn{4}{|l|}{ Tumour size } \\
\hline$<5 \mathrm{~cm}$ & $27 / 29(93.1 \%)$ & $20.21(2.96)$ & \multirow{2}{*}{$0.042^{\bigotimes}$} \\
\hline$\geq 5 \mathrm{~cm}$ & $17 / 24(70.8 \%)$ & $12.42(2.38)$ & \\
\hline \multicolumn{4}{|l|}{ Primary tumour } \\
\hline $\mathrm{T} 2$ & $10 / 12(83.3 \%)$ & $14.33(4.303)$ & \multirow{3}{*}{$0.120^{*}$} \\
\hline $\mathrm{T} 3$ & $9 / 10(90 \%)$ & $24.3(4.41)$ & \\
\hline $\mathrm{T} 4$ & $25 / 31(80.6 \%)$ & $15.129(2.56)$ & \\
\hline \multicolumn{4}{|l|}{ Tumour grade } \\
\hline Well differentiated & $22 / 25(88 \%)$ & $20.20(14.99)$ & \multirow{3}{*}{$0.035^{*}$} \\
\hline Moderately differentiated & $52 / 63(82.5 \%)$ & $16.95(14.39)$ & \\
\hline Poorly differentiated & $3 / 5(60 \%)$ & $3.6(4.62)$ & \\
\hline \multicolumn{4}{|l|}{ Nodal metastasis } \\
\hline Positive & $24 / 31(77.4 \%)$ & $14.19(13.11)$ & \multirow{2}{*}{$0.229^{\bigotimes}$} \\
\hline Negative & $22 / 24(91.7 \%)$ & $18.63(15.46)$ & \\
\hline \multicolumn{4}{|l|}{ Lymphovascular invasion } \\
\hline Positive & $6 / 7(85.7 \%)$ & $12.29(3.39)$ & \multirow{2}{*}{$0.729^{\bigotimes}$} \\
\hline Negative & $38 / 46(82.6 \%)$ & $16.913(2.22)$ & \\
\hline \multicolumn{4}{|l|}{ Margin status } \\
\hline Involved & $4 / 6(66.7 \%)$ & $20.33(7.63)$ & \multirow{2}{*}{$0.651^{\square}$} \\
\hline Free & $40 / 47(85.1 \%)$ & $15.79(2.04)$ & \\
\hline \multicolumn{4}{|l|}{ Recurrence } \\
\hline Recurrence & $27 / 30(90 \%)$ & $19.36(2.6)$ & \multirow[t]{2}{*}{$0.474^{\bowtie}$} \\
\hline No recurrence & 29/33 (87.9\%) & $16.8(2.58)$ & \\
\hline \multicolumn{4}{|l|}{ Status at end point } \\
\hline Died of disease & 21/23 (91.3\%) & $18.22(3.11)$ & \multirow{2}{*}{$0.748^{\bigotimes}$} \\
\hline Alive & $58 / 70(82.6 \%)$ & $16.74(1.74)$ & \\
\hline
\end{tabular}

${ }^{{ }}$The Mann-Whitney test; ${ }^{*}$ The Kruskal-Wallis test; SEM: standard error of the mean.

at the transcript level in HT29 colon adenocarcinoma cells [19].

In this study, we examined the expression and localisation of villin protein in a subset of CRC and a number of adjacent histologically normal mucosa. As reported before [8], we have described three patterns of cellular localisation of villin: apical membranous (brush border), cytoplasmic, and mixed pattern. In normal mucosa, $100 \%$ of examined samples showed villin immunoexpression with brush border localisation and a minor cytoplasmic element. In tumour samples, villin



Figure 2: Disease-free survival curve (Kaplan Meier) according to villin immunostaining; 0 : negative villin expression; 1 : low villin expression; 2 : moderate villin expression $(\log -\mathrm{rank}=6.604, P=$ 0.037).

was found to be expressed in $84.9 \%$ of cases; cytoplasmic localisation was detected in $100 \%$ of positive cases, while the characteristic brush border was detected in $70.9 \%$ of positive cases. Arango et al. also observed mislocalisation of villin CRC away from normal brush border localisation [6]. In our study, $29.1 \%$ of positive cases were lacking the characteristic brush border staining. Several authors have reported that villin brush border staining is exclusive for gastrointestinal adenocarcinoma $[8,13,20]$. However, villin expression was described in nongastrointestinal carcinomas such as renal cell carcinoma and endometrial carcinoma [9]. Also, abnormal localisation has been described in neuroendocrine carcinoma from different organs [10]. The mechanism of translocation of villin to the cytoplasm of malignant cells has not yet been clarified and needs to be addressed. We may speculate that villin production is required at different stages of epithelial cell transformation in CRC.

In our study, we demonstrated immunoexpression in CRC is downregulated than normal. This is reflected at two levels, the percentage of positive cells are less than normal (84.9\%). Second is that in CRC samples majority of positive cases are moderately expressing villin while in normal mucosa the majority showed high villin expression. This finding is consistent with findings of Werling et al. [21] and Arango et al. [6]. However, other investigators stated that villin had been shown to be expressed in almost $100 \%$ of primary and metastatic adenocarcinomas of the colon $[8,9,22,23]$. The difference may be explained by using different sample size, antibody clone, or technical consideration in immunohistochemistry. So, absence of villin 
expression in a metastatic poorly differentiated CRC does not exclude gastrointestinal origin [6]. Decrease in villin level has been described in pathological conditions of colon such as Crohn's disease and ulcerative colitis [24], and this is supported by finding that villin knockout mice have higher susceptibility to intestinal cell damage [4]. Villin expression is regulated by $\mathrm{Cdx}-2$ transcription factor which enhances villin expression [25]. Cdx-1 (transcriptional factor) is strongly correlated with villin expression. Villin downregulation in $\mathrm{CRC}$ is a consequence of loss of Cdx-1 expression; a likely possibility is that multiple proteins that are Cdx-1 targets will be downregulated in parallel and that collectively this results in a loss of glandular differentiation [6].

The relation between villin and clinicopathological characteristics has been addressed in our study. We could not establish association between villin immunoexpression and nodal metastasis. Also villin immunoexpression was not able to predict nodal metastasis. However in other studies, cells expressing villin migrate faster than villin-null cells [15] and villin may be modified during metastasis [7]. In addition, there was no statistically significant relation between villin expression and patients' age, tumour location, depth of invasion, lymphovascular invasion, margins, or recurrence.

We found also that villin expression was higher in welldifferentiated and moderately differentiated CRC than in poorly differentiated CRC, which is similar to what was found previously [6]. Bacchi and Gown showed that no relationship was found between the presence, or pattern of expression, of villin and the state of tumour differentiation [8]. Whether villin is preserved, downregulated, or lost completely in poorly CRC has to be verified by further studies due to small number of poorly differentiated samples in our study and previous studies.

In this study, villin immunoexpression in tumours with a size less than $5 \mathrm{~cm}$ in diameter was significantly higher than in those of $5 \mathrm{~cm}$ or more. This finding is genuine and has no comparable results. This means that villin may be involved in inhibiting tumour growth and proliferation. Villin is involved in regulation of actin dynamics, signal transduction, cell morphology, epithelial-to-mesenchymal transition, cell migration, cell invasion and cell survival in renal cell lines, intestinal cell lines, and villin-null mice [14, 15, 26-29]. Another contradictory finding in villin is an epithelial cellspecific antiapoptotic protein $[7,12]$. The exact mechanism behind this observation remains a matter for further research including proliferation assay and invasion assay.

We have found that CRC with higher villin immunoexpression are associated with better survival outcome. On the other hand, Arango et al. found no such association between villin staining intensity and overall survival in CRC [6].

Most current studies focus on using villin as a differentiation marker for metastatic gastrointestinal adenocarcinomas. Data is still lacking regarding the biological importance of villin immunoexpression in CRC and other tumours. We need to extend our knowledge in discovering the mechanistic pathways of villin in tumorigenesis.

Limitations of our study are incomplete follow-up data of some cases and small number of poorly differentiated CRC in samples examined.

\section{Conclusion}

In summary, villin was downregulated compared to normal counterpart. We have shown that villin immunoexpression in CRC is associated with better survival, well-differentiated tumours, and small-sized tumours. Villin has no significant association with disease recurrence or nodal metastasis. More in vivo and in vitro studies are required for further elucidation of how villin may be involved in CRC.

\section{Conflict of Interests}

The authors declare that they have no conflict of interests.

\section{Acknowledgment}

This work was supported by the Scientific Chair for Colorectal Cancer, King Abdulaziz University, Jeddah, Saudi Arabia.

\section{References}

[1] J. W. Huh, H. R. Kim, Y. J. Kim et al., "Expression of standard CD44 in human colorectal carcinoma: association with prognosis," Pathology International, vol. 59, no. 4, pp. 241-246, 2009.

[2] T. Kunimura, T. Yoshida, T. Sugiyama, and T. Morohoshi, “The relationships between loss of standard CD44 expression and lymph node, liver metastasis in T3 colorectal carcinoma," Journal of Gastrointestinal Cancer, vol. 40, no. 3-4, pp. 115-118, 2009.

[3] H. Al-Eid and S. Arteh, "Cancer Incidence Report Saudi Arabia," 1-99, 2005.

[4] R. Athman, D. Louvard, and S. Robine, "The epithelial cell cytoskeleton and intracellular trafficking III. How is villin involved in the actin cytoskeleton dynamics in intestinal cells?" American Journal of Physiology, vol. 283, no. 3, pp. G496-G502, 2002.

[5] A. Bretscher and K. Weber, "Villin is a major protein of the microvillus cytoskeleton which binds both $\mathrm{G}$ and $\mathrm{F}$ actin in a calcium-dependent manner," Cell, vol. 20, no. 3, pp. 839-847, 1980.

[6] D. Arango, S. Al-Obaidi, D. S. Williams et al., "Villin expression is frequently lost in poorly differentiated colon cancer," American Journal of Pathology, vol. 180, no. 4, pp. 1509-1521, 2012.

[7] S. Khurana and S. P. George, "Regulation of cell structure and function by actin-binding proteins: villin's perspective," FEBS Letters, vol. 582, no. 14, pp. 2128-2139, 2008.

[8] C. E. Bacchi and A. M. Gown, "Distribution and pattern of expression of villin, a gastrointestinal-associated cytoskeletal protein, in human carcinomas: a study employing paraffinembedded tissue," Laboratory Investigation, vol. 64, no. 3, pp. 418-424, 1991.

[9] R. Moll, S. Robine, B. Dudouet, and D. Louvard, "Villin: a cytoskeletal protein and a differentiation marker expressed in some human adenocarcinomas," Virchows Archiv B, vol. 54, no. 3, pp. 155-169, 1987.

[10] P. J. Zhang, K. R. Harris, B. Alobeid, and J. J. Brooks, "Immunoexpression of villin in neuroendocrine tumors and its diagnostic implications," Archives of Pathology and Laboratory Medicine, vol. 123, no. 9, pp. 812-816, 1999.

[11] V. Subramaniam, I. R. Vincent, H. Gardner, E. Chan, H. Dhamko, and S. Jothy, "CD44 regulates cell migration in human colon cancer cells via Lyn kinase and AKT phosphorylation," 
Experimental and Molecular Pathology, vol. 83, no. 2, pp. 207215,2007

[12] Y. Wang, K. Srinivasan, M. R. Siddiqui, S. P. George, A. Tomar, and S. Khurana, "A novel role for villin in intestinal epithelial cell survival and homeostasis," The Journal of Biological Chemistry, vol. 283, no. 14, pp. 9454-9464, 2008.

[13] N. Yoshino, H. Kubokura, S. Yamauchi, Y. Ohaki, K. Koizumi, and K. Shimizu, "Mucinous carcinoma identified as lung metastasis from an early rectal cancer with submucosal invasion by immunohistochemical detection of villin," Japanese Journal of Thoracic and Cardiovascular Surgery, vol. 54, no. 8, pp. 328-331, 2006.

[14] R. Athman, M.-I. Fernandez, P. Gounon et al., "Shigella flexneri infection is dependent on villin in the mouse intestine and in primary cultures of intestinal epithelial cells," Cellular Microbiology, vol. 7, no. 8, pp. 1109-1116, 2005.

[15] A. Tomar, Y. Wang, N. Kumar et al., "Regulation of cell motility by tyrosine phosphorylated villin," Molecular Biology of the Cell, vol. 15, no. 11, pp. 4807-4817, 2004.

[16] N. Al-Muhannadi, N. Ansari, U. Brahmi, and A. A. Satir, "Differential diagnosis of malignant epithelial tumours in the liver: an immunohistochemical study on liver biopsy material," Annals of Hepatology, vol. 10, no. 4, pp. 508-515, 2011.

[17] S. K. Lau, S. Prakash, S. A. Geller, and R. Alsabeh, "Comparative immunohistochemical profile of hepatocellular carcinoma, cholangiocarcinoma, and metastatic adenocarcinoma," Human Pathology, vol. 33, no. 12, pp. 1175-1181, 2002.

[18] H.-J. Grone, K. Weber, U. Helmchen, and M. Osborn, "Villina marker of brush border differentiation and cellular origin in human renal cell carcinoma," American Journal of Pathology, vol. 124, no. 2, pp. 294-302, 1986.

[19] E. Pringault, M. Arpin, A. Garcia, J. Finidori, and D. Louvard, "A human villin cDNA clone to investigate the differentiation of intestinal and kidney cells in vivo and in culture," EMBO Journal, vol. 5, no. 12, pp. 3119-3124, 1986.

[20] Y. Nambu, M. D. Iannettoni, M. B. Orringer, and D. G. Beer, "Unique expression patterns and alterations in the intestinal protein villin in primary and metastatic pulmonary adenocarcinomas," Molecular Carcinogenesis, vol. 23, pp. 234-242, 1998.

[21] R. W. Werling, H. Yaziji, C. E. Bacchi, and A. M. Gown, "CDX2, a highly sensitive and specific marker of adenocarcinomas of intestinal origin: an immunohistochemical survey of 476 primary and metastatic carcinomas," American Journal of Surgical Pathology, vol. 27, no. 3, pp. 303-310, 2003.

[22] J. Tan, G. Sidhu, M. A. Greco, H. Ballard, and R. Wieczorek, "Villin, cytokeratin 7, and cytokeratin 20 expression in pulmonary adenocarcinoma with ultrastructural evidence of microvilli with rootlets," Human Pathology, vol. 29, no. 4, pp. 390-396, 1998.

[23] A. B. West, C. A. Isaac, J. M. Carboni, J. S. Morrow, M. S. Mooseker, and K. W. Barwick, "Localization of villin, a cytoskeletal protein specific to microvilli, in human ileum and colon and in colonic neoplasms," Gastroenterology, vol. 94, no. 2, pp. 343-352, 1988.

[24] S. Kersting, M. Bruewer, G. Schuermann et al., "Antigen transport and cytoskeletal characteristics of a distinct enterocyte population in inflammatory bowel diseases," American Journal of Pathology, vol. 165, no. 2, pp. 425-437, 2004.

[25] N. Yamamichi, K.-I. Inada, C. Furukawa et al., "Cdx2 and the Brm-type SWI/SNF complex cooperatively regulate villin expression in gastrointestinal cells," Experimental Cell Research, vol. 315, no. 10, pp. 1779-1789, 2009.
[26] R. A. D. Louvard and S. Robine, "Villin enhances hepatocyte growth factor-induced actin cytoskeleton remodeling in epithelial cells," Molecular Biology of the Cell, vol. 14, no. 11, pp. 46414653, 2003

[27] S. Khurana, A. Tomar, S. P. George et al., "Autotaxin and lysophosphatidic acid stimulate intestinal cell motility by redistribution of the actin modifying protein villin to the developing lamellipodia," Experimental Cell Research, vol. 314, no. 3, pp. 530-542, 2008.

[28] A. Tomar, S. George, P. Kansal, Y. Wang, and S. Khurana, "Interaction of phospholipase C- $\gamma 1$ with villin regulates epithelial cell migration," The Journal of Biological Chemistry, vol. 281, no. 42, pp. 31972-31986, 2006.

[29] Y. Wang, A. Tomar, S. P. George, and S. Khurana, "Obligatory role for phospholipase C-gamma(1) in villin-induced epithelial cell migration," American Journal of Physiology, vol. 292, pp. C1775-C1786, 2007. 


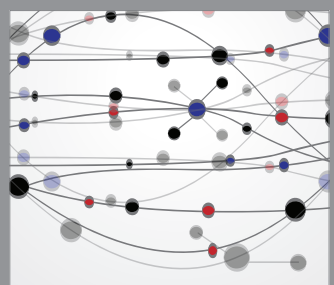

The Scientific World Journal
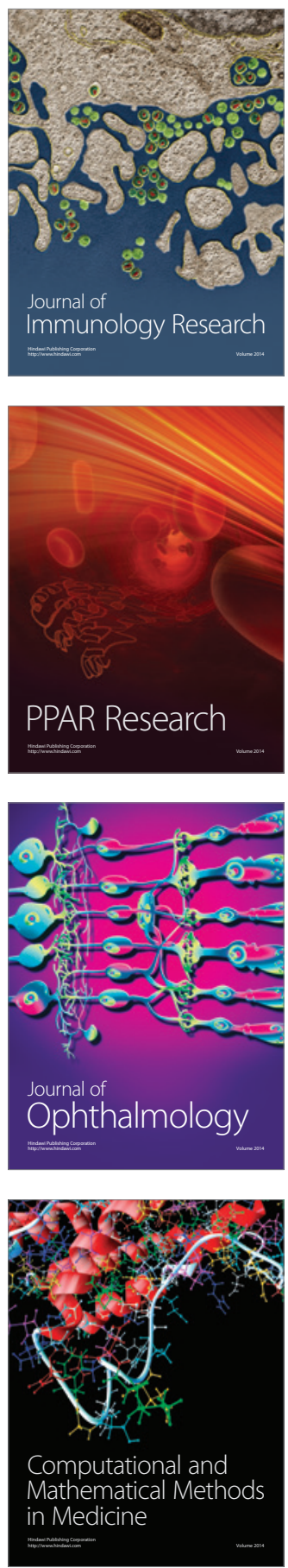



Gastroenterology

Research and Practice
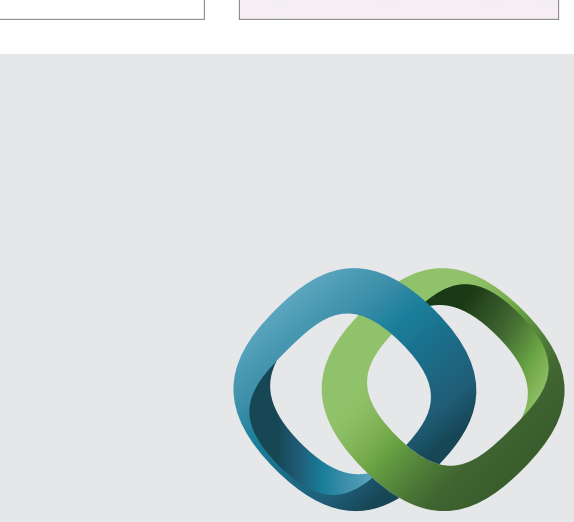

\section{Hindawi}

Submit your manuscripts at

http://www.hindawi.com
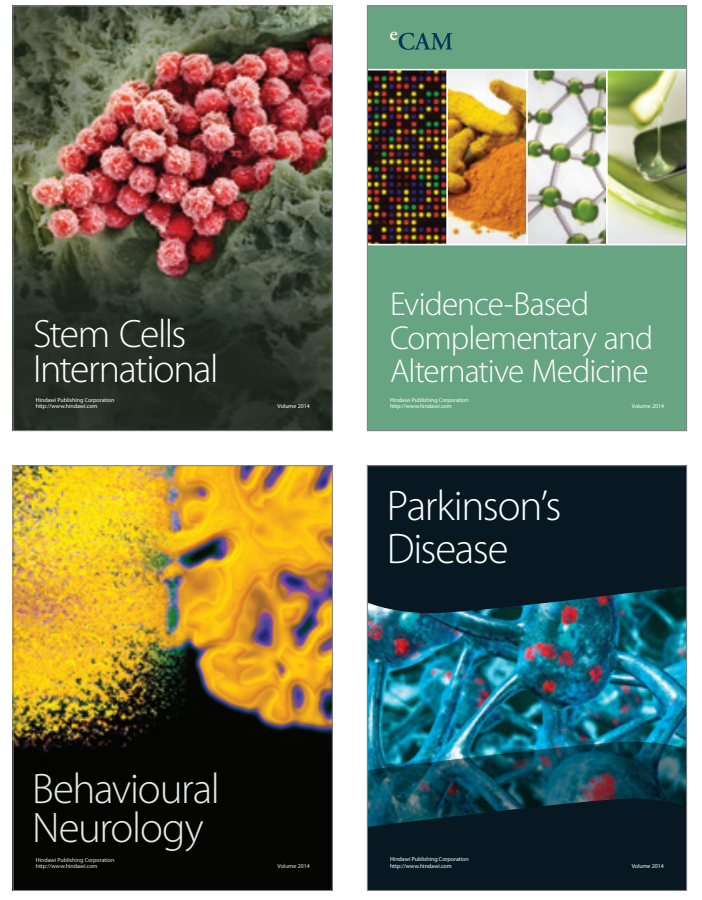
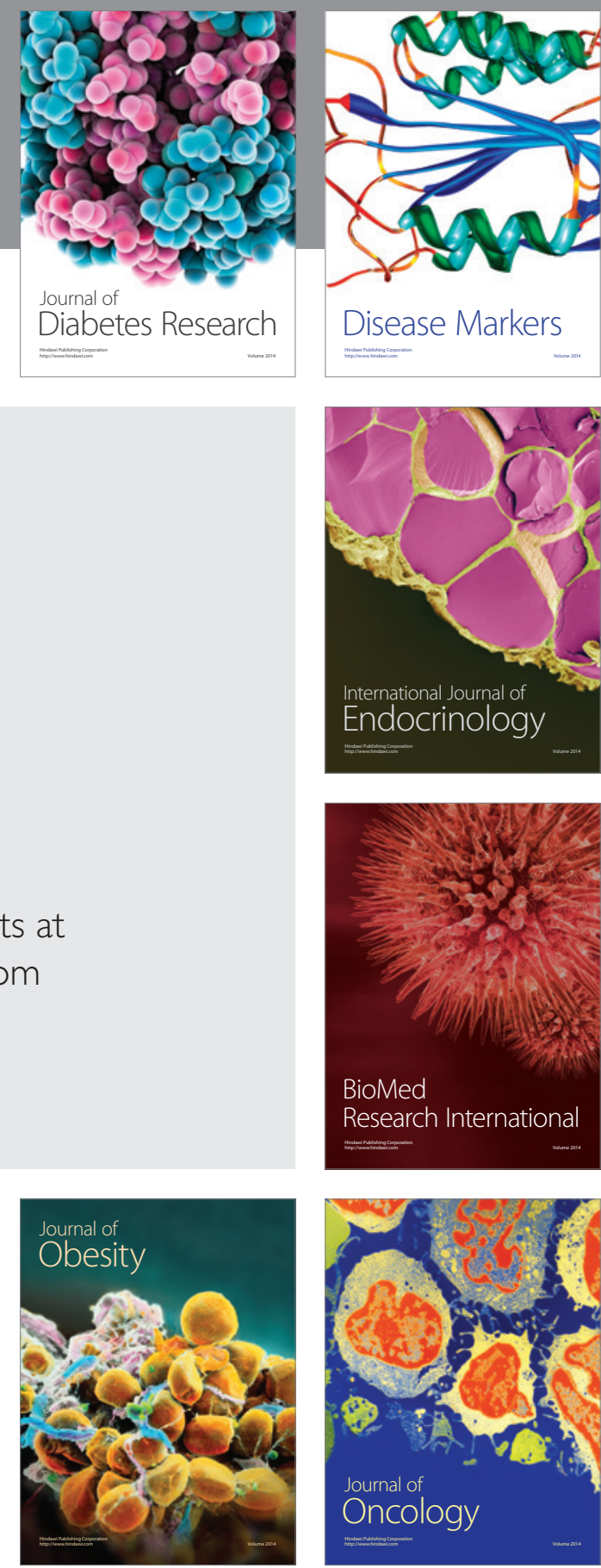

Disease Markers
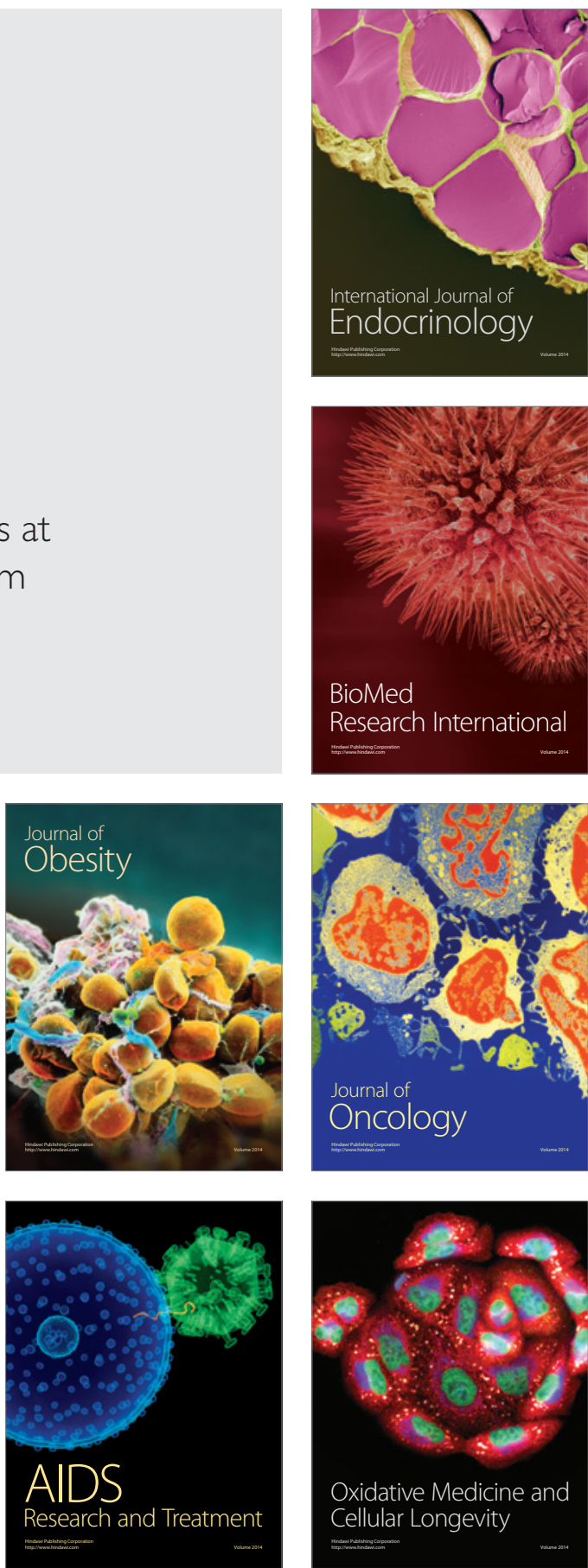